colleagues find that more than half of the species in a given cell (and almost $90 \%$ in most marine assemblages) tend to have geographical ranges that encompass similarly warm temperatures, such that they would all face exposure at around the same time.

Such a striking pattern of shared thermal niches within assemblages has been observed before, in a global analysis of marine fishes and invertebrates ${ }^{4}$. In that study, species' thermal niches were found not to change gradually with latitude, but instead to have distinct transition points, indicating that species belong to what are termed thermal guilds ${ }^{4}$. These shared thermal niches could be due to physical boundaries or ecological interactions that restrict the ranges - and temperatures experienced - of multiple species similarly. Or this phenomenon might be the result of a low rate of evolution in the range of temperatures across which the species can fundamentally persist, leading to the maintenance of thermal guilds.

When does this abrupt exposure happen? It is predicted that it will occur at different times for grid cells around the world, from some predicted to be occurring already in the ocean, to others occurring towards the end of the projected time range, in 2100 . That the timing is different across grid cells is a good thing, because at least all of the assemblages aren't predicted to experience abrupt losses at the same time. But, notably, the timing of exposure does not correlate with the timing of climate-change emergence in temperature, suggesting that the latter metric might be a poor predictor of major biodiversity change within a given grid cell.

Trying to project the timing of biodiversity shifts is a noble objective that will surely help us to develop management systems and anticipate crises. Although Trisos et al. provide an initial approach that offers useful insights, further studies should attempt to validate and qualify these predictions. For example, Trisos and colleagues used temperatures outside species' current thermal niches to define climate exposure, but we don't know what will really occur when species experience such temperatures - many can certainly tolerate temperatures beyond those found in their current ranges ${ }^{5,6}$. The timing of exposure to truly limiting environments might turn out to be more diverse across species than currently predicted by Trisos et al. if variation in species' fundamental climatic niches (the range of temperatures and other climate variables across which an organism can survive) is considered. It will also be useful to consider the flip side of the range-shift issue: the timing and abruptness with which new species enter an assemblage as a result of range extensions arising from climate change.

Most crucially, as climate change progresses, we should be able to test and refine projections such as these using real-time observations. Where are biodiversity changes already occurring abruptly? The need for systematic global biodiversity monitoring has never been stronger.

Jennifer M. Sunday is in the Department of Biology, McGill University, Montreal, Quebec H3A 1B1, Canada.

e-mail: jennifer.sunday@mcgill.ca
Warren, R., Price, J., Graham, E., Forstenhaeusler, N. \& VanDerWal, J. Science 360, 791-795 (2018).

2. Trisos, C. H., Merow, C. \& Pigot, A. L. Nature 580, 496-501 (2020).

3. Harris, R. M. B. et al. Nature Clim. Change 8, 579-587 (2018). 4. Stuart-Smith, R. D., Edgar, G. J., Barrett, N. S. Kininmonth, S. J. \& Bates, A. E. Nature 528, 88-92 (2015).

5. Sunday, J. M., Bates, A. E. \& Dulvy, N. K. Nature Clim. Change 2, 686-690 (2012).

6. Early, R. \& Sax, D. F. Glob. Ecol. Biogeogr. 23, 1356-1365 (2014)

7. van Vuuren, D. P. et al. Clim. Change 109, 5-31(2011).

This article was published online on 8 April 2020.

\title{
Evolution
}

\section{Can't see the wood for the trees}

\section{Mark Pagel}

Evolutionary-tree diagrams, which show the branching

relationships between species, are widely used to estimate

the rates at which new species arise and existing ones become

extinct. New work casts doubt on this approach. See p.502

Scientists often want to make inferences about what the biological past was like, and how that past gave rise to the present, because doing so allows them to understand the processes that drive evolution. But on page 502, Louca and Pennell ${ }^{1}$ challenge a major aspect of that enterprise.

Specifically, their work regards the issue of estimating past rates of speciation and extinction, which are, respectively, the rates at which new species arise and existing species go extinct. These rates determine the number of contemporary species of various forms. There are, for instance, around 6,600 species

\section{“Assumptions are being made about the things that we would like to estimate."}

of songbird (passerines), which constitute more than half of all existing bird species, and we might therefore be tempted to say that songbirds have a high rate of speciation in comparison with that of other birds. But it's also possible to speculate that they have a low extinction rate. Louca and Pennell show that the uncertainty is even worse than this: not only can we not estimate these two rates, but also there is an infinite number of different sets of these two parameters that are equally good at describing any particular outcome, such as the number of species of contemporary songbird.

Because fossils are scarce or non-existent for the vast majority of species, evolutionary scientists instead estimate speciation and extinction rates from phylogenies - tree diagrams that describe the patterns of descent among a group of contemporary species (Fig. 1a,b). For any such phylogeny, it is easy to construct what is termed a lineage-through-time plot; this records the cumulative number of lineages up to that point in time on the tree that will eventually leave one or more living descendent species (Fig.1c). The slope of the curve fitted to such a plot, often denoted by $\lambda$, is the net speciation rate. This is equal to the difference between the rate of speciation, termed $b$ (or birth), and the rate of extinction, termed $d$ (or death). It is described by the equation $\lambda=b-d$.

However, it is known that a difficulty arises in estimating $b$ and $d$, because if all that is available is the number of species that have survived to the present, such as our 6,600 songbirds, any pair of $b$ and $d$ that returns the same value of $\lambda$ will produce an identical lineage-through-time curve, and there is an infinite number of these pairs. In fact, it turns out that for the simple case of estimating $b-d$, such as described here, a feature of the shape of the lineage-through-time curve can be exploited to estimate the rate of extinction, and then the rate of speciation can be found by subtraction ${ }^{2}$. But to do so requires making the assumption that both of these rates are constant throughout the entire time span of the tree, when instead they almost certainly vary between the different branches (lineages) of the phylogeny, and through time.

This is where Louca and Pennell step in, 
a

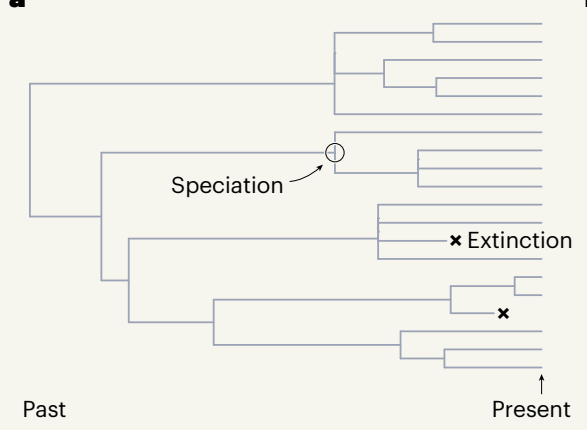

b

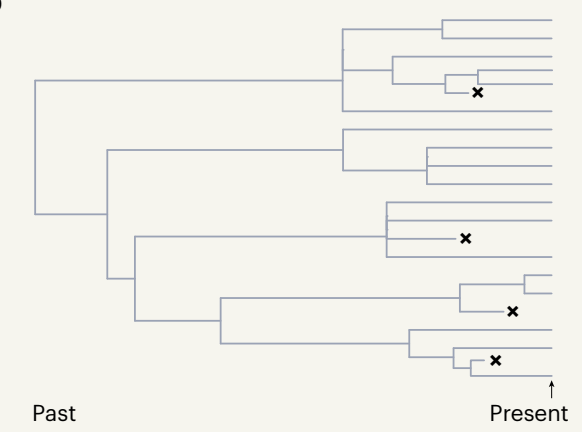

c

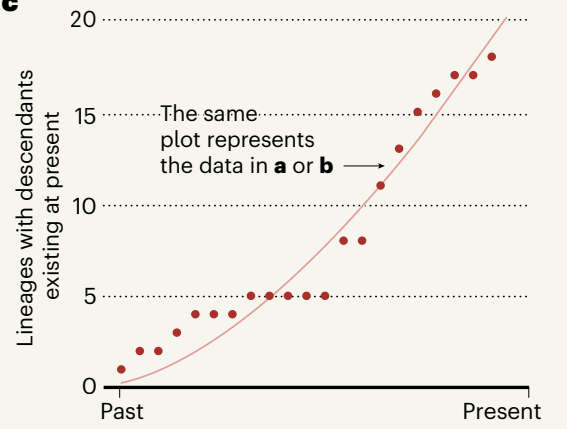

Figure $1 \mid$ Assessing evolutionary histories. Louca and Pennell ${ }^{1}$ raise questions about a standard approach to estimating past rates of species formation (speciation) and extinction that uses data from a lineage-through-time plot. The number of species in the present depends on how speciation and extinction rates varied over time in the past. Using mathematical modelling, the authors reveal that an infinite number of pairs of speciation and extinction rates could give rise to any given outcome, and it is thus unclear how to determine the correct rates. a, b, Examples of known extinctions are rare, and are shown in these hypothetical tree diagrams only to illustrate how different rates of extinction (and different speciation rates) can yield the same lineage-through-time plot. c, Information taken from a tree diagram can be represented in a lineage-through-time plot as shown. Red dots indicate the number of lineages at a given time that gave rise to lineages existing in the present. The slope of the curve equals the speciation rate minus the extinction rate. This plot is valid for both trees even though they have different speciation and extinction rates. This underscores the authors' demonstration that many different data inputs can give identical lineage-through-time plots. because the novelty and mathematical sophistication of their work lie in showing that we cannot estimate these 'time-varying' speciation and extinction rates. The authors invoke earlier work ${ }^{3}$ that defines the existence of a tree's 'deterministic' lineage-through-time curve: this is a set of differential equations (equations describing rates of change) that fully determine the number of lineages in a tree at any given time. Louca and Pennell's key result is then to show that there is an infinite number of alternative sets of time-varying speciation-extinction rates that yield the same number of lineages at any given time as does the deterministic lineage-through-time curve. They further show that the most probable estimates of the two rates (calculated by maximum-likelihood methods) do not necessarily identify the correct underlying model - as demonstrated by an analysis of hypothetical cases for which the true time-varying speciation-extinction rates are known.

Even worse for those who want to use the rates of speciation and extinction to study evolution, the possible alternative scenarios of time-varying speciation and extinction rates that are consistent with the deterministic lineage-through-time model often differ qualitatively. For example, the authors show that a phylogeny of approximately 80,000 species of seed plant is equally well described by speciation and extinction rates that both gradually increase through time or that both gradually decrease through time. Other scenarios, including rates that vary wildly with time, provide equally good descriptions of the numbers of lineages through time as derived from the deterministic lineage-through-time model.

Louca and Pennell's conclusions will be dispiriting to evolutionary scientists who are looking for a link between past levels of speciation and extinction and historical climate change or other environmental events, or who want to test ideas about what features of a species - such as diet, mating system or the length of a generation - might be used to predict speciation and extinction rates ${ }^{4}$. The limitations that Louca and Pennell have identified for estimating speciation and extinction rates do not go away as the size of the phylogenetic tree increases. Nor do other common features of trees provide much help: for example, if a group of species has never suffered any extinctions, estimating their speciation rate would be straightforward. But this is rare, and unlikely to be known in advance. Having abundant fossils could help, because they provide evidence needed to estimate extinction rates; however, fossils are seldom abundant. We can make assumptions about how speciation and extinction might vary with each other, through time, or with the number of species, but these assumptions are being made about the things that we would like to estimate.

Amid this epistemological carnage regarding what we can possibly know, the authors helpfully offer some consolation by showing that it is possible to estimate a parameter they call the pulled speciation rate, or $\lambda_{\mathrm{p}}$. This measures the rate of change (the slope of the curve) of the deterministic model of the lineage-through-time plot. The pulled speciation rate can be compared between lineages, or at different times, and might be useful for understanding the processes that gave rise to the species that are alive today, even if not necessarily providing information about those species that didn't make it.

And this aspect - the ones that became extinct - is the deeper lesson of Louca and
Pennell's work. Without fossils, all evolutionary scientists, whether studying speciation and extinction or attempting to reconstruct the features of distant ancestors, need to be aware that the evolutionary processes they identify are those that operated in the species that would survive and eventually leave descendants in the present. We can't be sure what was going on in those that went extinct. It is the evolutionary version of the observation that history is written by the victors. The supreme irony of this predicament is that Charles Darwin's idea about the survival of the fittest, the story that we want to understand, by its very nature renders elusive some of the key components needed to study it.

Mark Pagel is at the School of Biological Sciences, University of Reading, Reading RG6 6UR, UK. e-mail:m.pagel@reading.ac.uk
1. Louca, S. \& Pennell, M. W. Nature 580, 502-505 (2020).

2. Nee, S., Holmes, E. C., May, R. M. \& Harvey, P. H. Phil. Trans. R. Soc. Lond. B 344, 77-82 (1994).

3. Kubo, T. \& Iwasa, Y. Evolution 49, 694-704 (1995). 4. Morlon, H. Ecol. Lett. 17, 508-525 (2014). 